\title{
INCORPORATING MRI REGION INFORMATION INTO SPECT RECONSTRUCTION USING JOINT ESTIMATION
}

\author{
Yong Zhang, Jeffrey A. Fessler, Neal H. Clinthorne, W. Leslie Rogers \\ 3480 Kresge III, Box 0552 \\ The University of Michigan \\ Ann Arbor, MI 48109-0552 \\ zhg@engin.umich.edu
}

\begin{abstract}
Single Photon Emission Computed Tomographic images(SPECT) have relatively poor resolution. In an attempt to improve SPECT image quality, many methods have been developed for including anatomic information, extracted from higher resolution, structurally correlated Magnetic Resonance images(MRI), into SPECT reconstruction process. These methods provide improved SPECT reconstruction accuracy if the anatomic information is perfectly correlated with the SPECT functional information. However there exist mismatches between MRI anatomical structures and SPECT functional structures due to different imaging mechanisms. It has been reported that if the MR structures are applied into SPECT, the mismatched part will cause artifacts. This paper describes a joint estimation approach which unifies MR information extraction and SPECT reconstruction processes to avoid these artifacts. Both qualitative and quantitative evaluations show that the method improves the SPECT reconstruction where the MR information matches and is robust to mismatched MR information.
\end{abstract}

\section{INTRODUCTION}

SPECT has the capability of disclosing the functional information in living organs. However, the fluctuation of photon statistics, trade-offs between detection sensitivity and collimator resolution, and limited photon rates cause the inherently modest resolution in SPECT imaging. Since MRI give relatively high resolution images, many efforts have been made to apply region information, extracted from high resolution, structurally correlated MR images, to constrain the SPECT reconstruction as an image prior in a Maximum a posteriori (MAP) objective to improve the image quality and quantification. Two major approaches exist for incorporating structural information: line site models, which represent anatomic boundaries [2]; and labelling methods, which identify anatomic regions [4]. For example, one can define the region labels with preprocessed MRI and define the labels which define the weights in a weighted Gibb's function as a penalty function in a penalized Maximumlikelihood objective [3], so intensity discrepancies are only

This work was supported in part by NIH Grant CA54362 and DOE grant DE-FG02-87ER6056 penalized within the same region and not across region boundaries. This is a spatially-variant regularization. This provides improved SPECT reconstruction if the anatomical structure is perfectly correlated with SPECT functional information. However, it has been reported that this type of "blind" use of MRI side information causes artifacts [3] if there is mismatch between MRI anatomic and SPECT functional structures. In practice, this type of mismatch is probable since the spin density and $T_{1}, T_{2}$ distributions are not necessarily the same as SPECT tracer distribution. Some investigations have been done to accommodate this fact, such as Leahy's "functional line sites method" [1] and Fessler's "blurred weights" method [3]. To avoid fixed influence from the mismatched MRI structure, it is better to allow the line sites or region labels to vary. Thus we investigate a joint estimation approach that incorporates both the anatomic side information and its measurement statistics into SPECT image reconstruction. We choose region labels to represent the anatomical regions extracted from MRI into SPECT reconstruction because the region labels more easily favor region contiguity and need half as many the parameters as line sites. In this approach, each SPECT pixel corresponds to one region label. If two pixels have the same type of region labels, they tend to have the similar intensities. The region labels 1 are not simply a "copy" from the segmented MRI. After being initialized with the segmented MRI, they are jointly estimated with SPECT pixel mean intensity parameters $\lambda$, using a penalized MaximumLikelihood(PML) objective. We update a SPECT mean intensity pixel and its corresponding region label simultaneously, such that label-pixel pair updating are mutually constrained. Since region labels are estimated jointly from both MRI and SPECT, only those parts of the MR anatomical regions that match the SPECT functional regions are represented by the estimated labels, and constrain the SPECT intensity reconstruction, while the mismatched region labels will be updated during the joint estimation process, to approach the SPECT functional region. Thus the artifacts due to mismatch are reduced.

Section 2 describes the method in detail. Section 3 gives simulation results comparing visual qualities of the reconstructed images and bias vs. variance trade-offs of different methods. Some problems we encountered and possible future study are described in Section 4. 


\section{JOINT ESTIMATION APPROACH}

\subsection{Objective Function}

We choose penalized Maximum-Likelihood as our objective, which can also be viewed as a Maximum a posteriori if one regards the penalty terms as an $a$ priori pdf of possible image and label states from a Bayesian perspective. The joint objective function incorporates both the SPECT noise distribution and the MRI side information measurement statistics, plus a joint penalty function, as:

$$
\boldsymbol{\Phi}_{\text {joint }}(\lambda, \mathbf{l})=L(\lambda, \mathbf{l} ; \mathbf{y}, \tilde{\mathbf{l}})-\Omega(\lambda, \mathbf{l})
$$

where $y$ is the SPECT projection data, $\tilde{1}$ is the MR anatomic region measurement, $\lambda$ and $l$ are SPECT intensity and label parameters to be estimated, $L$ and $\Omega$ are joint likelihood and joint penalty terms. So the estimation of both SPECT intensity $\lambda$ and region labels $l$ will be:

$$
(\hat{\lambda}, \hat{1})=\arg \max _{\lambda, 1} \Phi_{j o i n t}(\lambda, 1) .
$$

\subsubsection{Joint Log-Likelihood $L(\lambda, \mathbf{1} ; \mathbf{y}, \tilde{1})$}

Since SPECT and MRI are independent imaging processes, their joint log-likelihood functions are independent and can be separated: $L(\lambda, \mathbf{l} ; \mathbf{y}, \tilde{\mathbf{1}})=L(\lambda ; \mathbf{y})+L(\mathbf{1} ; \tilde{1})$.

The SPECT measurement is well known to be Poisson process; the log-likelihood is given by [6]:

$$
L(\lambda, y)=\log f(\mathbf{y} ; \lambda) \equiv \sum_{n}\left(-\bar{y}_{n}(\lambda)+y_{n} \log \bar{y}_{n}(\lambda)\right)
$$

where

$$
\bar{y}_{n}(\lambda)=\sum_{k} a_{n k} \lambda_{k}+r_{n}
$$

where “” represents the equivalence, $r_{n}$ is the assumed known scatter. The log-likelihood of the region label variables, $L(1 ; \tilde{1})$ depends on how the anatomical information $\tilde{1}$ is extracted from the MR images. In our preliminary studies, we only concentrate on dealing with MRI mismatched structure, so we assume that there is no registration and distortion error in MRI. For simplicity, we also assume that the MRI has Gaussian noise and different regions have different intensities. If MRI is segmented using simple pixel-by-pixel thresholds, then the log-likelihood for $P\left(\hat{l}_{k} \mid l_{k}\right)$ for $\hat{l}_{k}$ given $l_{k}$ is:

$$
L(\mathbf{1} ; \tilde{\mathbf{1}})=\sum_{k=1}^{p} \log P\left(\tilde{l}_{k} \mid l_{k}\right)
$$

where $P\left(\tilde{l}_{k} \mid l_{k}\right)$ is the probability of assigning the label $\tilde{l}_{k}$ to the $k$ th pixel during segmentation when $l_{k}$ is the true label. Besides, the $P\left(\tilde{l}_{k} \mid l_{k}\right)$ also incorporates the possibility of existing mismatch in the segmented MRI. For example, in Fig.1, $P\left(\tilde{l}_{k}=B \mid l_{k}=C\right)$ can be determined by MRI noise statistics, however, whether there is a possible region $D$ in true region has to be decided by inspecting the segmented MRI and its corresponding. FBP, and then assign certain probability value. These values determine the extent to which we trust each region in the MRI structural information.

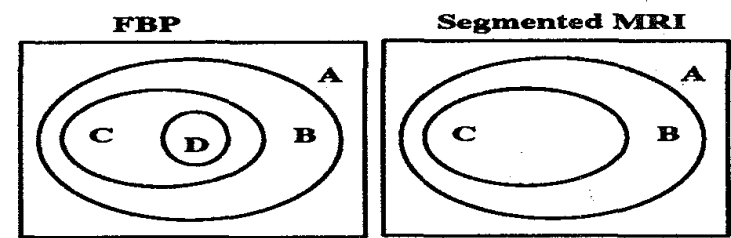

Figure 1: Compare FBP and MRI for $P\left(\tilde{l}_{k} \mid l_{k}\right)$ where probable mismatch exists.

\subsubsection{Joint Penalty $\Omega(\lambda, 1)$}

The joint penalty term $\Omega(\lambda, 1)$ can be viewed as a log-prior pdf of the SPECT pixels and region labels. We adopt a hierarchical form of $\Omega$, thus

$$
\Omega(\lambda, 1)=\beta_{1} \Omega_{1}(\lambda ; \mathbf{l})+\beta_{2} \Omega_{2}(\mathbf{l})
$$

where $\beta_{1}$ and $\beta_{2}$ are the penalty parameters. The penalty terms restrict the possible space of SPECT image and region labels. $\Omega_{1}$ relates the estimation of the labels 1 to the estimation of the intensity $\lambda$, and $\Omega_{2}$ is a penalty term which encourages contiguous regions. To encourage smoothness within the same region but allow discontinuities between regions in the SPECT image, we choose $\Omega_{1}$ as:

$$
\Omega_{1}(\lambda, 1)=\frac{1}{2} \sum_{k=1}^{p} \sum_{j \in \mathcal{N}_{k}} \omega_{k j}(1) \psi\left(\lambda_{k}-\lambda_{j}\right)
$$

with $\omega_{k j}(\mathbf{l})= \begin{cases}1 \text { (direct neighbor) } & \text { if } l_{k}=l_{j} \\ \sqrt{2} / 2 \text { (diagonal neighbor) } & \text { if } l_{k}=l_{j} \\ 0 & \text { if } l_{k} \neq l_{j}\end{cases}$

$\mathcal{N}_{k}$ is the neighborhood of pixel $k$, usually a 2nd-order neighborhood. The weights $\omega_{k j}$ link the pixel $k$ with its neighbors $j$, and $\psi\left(\lambda_{k}-\lambda_{j}\right)$ is usually a quadratic term. Here label $l$ are variables to be estimated. When $\psi\left(\lambda_{k}-\lambda_{j}\right)$ is too big, the minimization of the penalty pushes the current label $l_{k}$ to be different from its neighbors. This penalty term associates the SPECT intensity vector with the label vector, their estimations are affected by each other, instead of letting the labels extracted from MR dominate the SPECT reconstruction. We choose $\Omega_{2}$ as:

$$
\Omega_{2}(\mathrm{l})=\sum_{k=1}^{p} \sum_{j \in \mathcal{N}_{k}} \omega_{k j}(\mathrm{l})
$$

which encourages the same labels for neighboring pixels.

\subsection{Reconstruction Algorithm}

We apply an iterative Space-Alternating Generalized EM (SAGE) [5] algorithm for the penalized Maximum-Likelihood objective because of its faster convergence and its monotonicity. In SAGE, the penalized likelihood $\boldsymbol{\Phi}_{\text {joint }}(\lambda, 1)$ is maximized by maximizing $\phi^{k}\left(\lambda_{k}, l_{k} ; \lambda^{i}, 1^{i}\right)$, which is the sum of the conditional expectation $Q^{k}\left(\lambda_{k}, \lambda^{i}\right)$ of the loglikelihood and the penalty terms of the hidden-data space [5]. Each pixel-label pair is jointly updated by holding the remaining pixel-label pairs fixed:

$$
\left(\lambda_{k}^{i+1}, l_{k}^{i+1}\right)=\arg \max _{\left(\lambda_{k}, l_{k}\right)} \phi^{k}\left(\lambda_{k}, l_{k} ; \lambda^{i}, 1^{i}\right)
$$




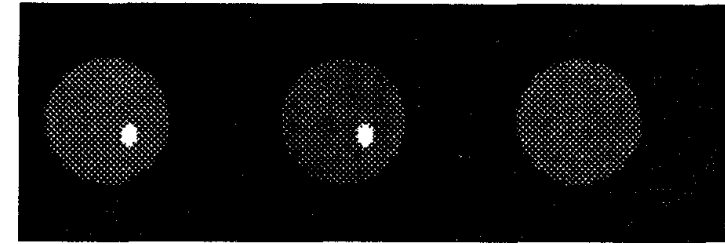

Figure 2: Top(a): The phantom of SPECT tracer distribution. Middle(b): Simulated segmented MRI region map with perfectly matching information. Bottom(c): Simulated segmented MRI region map with mismatched side information (a missing hot elliptical region).

SAGE algorithms monotonically increase both $\phi^{k}$ and $\boldsymbol{\Phi}_{\text {joint }}(\lambda, 1)$. However the penalty term $\Omega_{1}$ contains mixed discrete and continuous variables, the objective function is non-concave, so the SAGE here does not guarantee global convergence. To avoid local maxima, we use a deterministic annealing procedure by changing $\beta_{2}$ exponentially as a function of iteration number until a specified value is reached.

\section{SIMULATION RESULTS}

Some preliminary simulations are performed to compare the results from the joint estimation method and other image reconstruction methods in terms of both visual quality and bias vs. variance relationship. A simple parallel collimated SPECT imaging system is simulated, with a 46-bin detector and 90 sampling angles over the 180 -degree range. The image field is discretized into $64 \times 64$ pixels. The bin width is 2-pixel, and the detector response is triangular with 4-pixel FWHM. We ignore the attenuation. All of each SPECT projection sets have about 500,000 counts with $15 \%$ assumed known scatter, Poisson noise is simulated. Figure 2 are the phantoms used in these simulations.

Figure 3 shows images reconstructed in methods other than joint estimation. (a) is a filtered-backprojection with a 3rd-order Butterworth window, at $f_{c}=.80$. Fig.3(b) through Fig.3(d) are from penalized SAGE with weighted Gibbs quadratic penalty. (b) is without any anatomical information, using uniformly weighted Gibbs quadratic penalty. In order to reduce noise, the image has to be globally regularized, so it looks blurry across all the region boundaries. In (c) the Gibbs weights were defined by the perfect MRI anatomical regions, Fig.2(b). Thus the regions are smooth, and boundaries are sharp. This shows the ultimate (also unrealistic) performance of applying MRI side information. In Fig.3(d), Fig.2(c) was used as side information to define the weights in the penalty term. Where the anatomical regions match the SPECT functional region, the reconstruction is as good as Fig.3(c); where mismatch exists, then the hot region in SPECT is blurred about the same amount as in Fig.3(b) where the penalty is uniformly weighted.

For joint estimation, penalized SAGE is applied to update each pixel-label pair $\left(\lambda_{k}, l_{k}\right)$ in four alternative raster scan orders. An idealized $L(1 ; \tilde{\mathbf{1}})$ table is built and used according to the assumptions and procedure described in section 2 , see Table I. $\lambda$ is initialized with ramp filtered FBP reconstruction result, and $l$ is initialized with Fig.2(c)

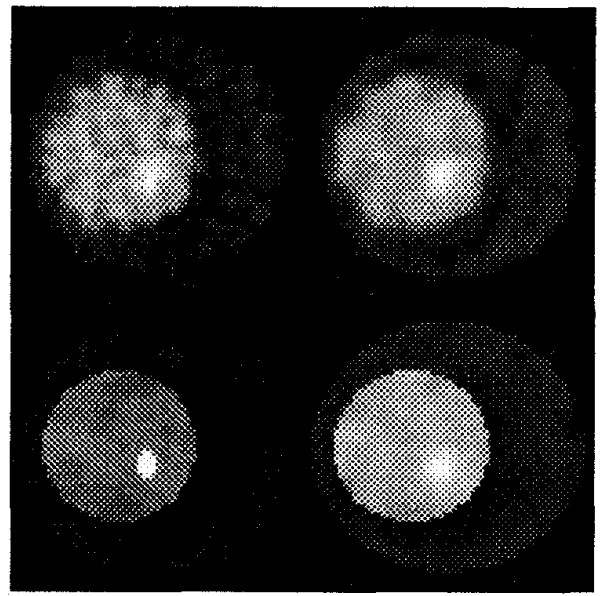

Figure 3: $\quad$ Top row:(a:left)FBP reconstruction. (b.right):SAGE reconstruction with uniform 8 neighbor regularization. Bottom row:(c:left)SAGE with perfect MRI anatomical region information. (d:right)SAGE with mismatched MRI anatomical information.

which represents mismatched MRI anatomical information. The reconstruction results are shown as Fig.4(a) and (b). We use fixed $\beta_{1}=2^{-9}$ and an exponentially increased $\beta_{2}=2^{0.5} \times 2^{\text {min(3,iteration })}$ for deterministic annealing process in the penalty terms. Even though there was no region information associated with the hot region, the joint estimation partially recovers the labels for that region and helps to prevent the blurring observed in both Fig.3(b) and Fig.3(d). The newly estimated region labels are basically dependent on SPECT data, so they are not the same as the true region labels, due to the noise in SPECT data.

To avoid anecdotal performance comparison and to observe the improvement in quantification, we generated 50 SPECT noisy projection realizations and evaluated the bias vs. standard deviation (std) trade-offs for the hot region in terms of its concentration among different methods. For the cases where the perfect MRI side information is available, we use the ideal ROI labels to define the region. For joint estimation with mismatched MRI side information, we use estimated labels to define that region. For uniform regularization and fixed application of MRI cases, we use $75 \%$ of the peak value in a bigger region containing the ROI as a threshold to define the estimated ROI. The concentration is then the averaged counts per pixel in that defined region. Fig. 5 shows the bias vs. STD comparison. When the perfectly matched MR anatomical information is used to define the weights of the penalty, it gives ultimate performance, i.e. for a given standard deviation, it has the smallest bias (case 1). On the contrary, when there is no MRI side information, we can only apply uniform regularization to control the trade-off between image resolution and noise. For given standard deviation, this gives the highest bias(case 2), and vice versa. Very similar performances are observed in filtered-backprojections with 3rd-order Butterworth window(Case 3 ) at $f_{c}=0.3,0.5,0.7$ and 0.9 and a Wiener filter which incorporates the triangular detector response at 


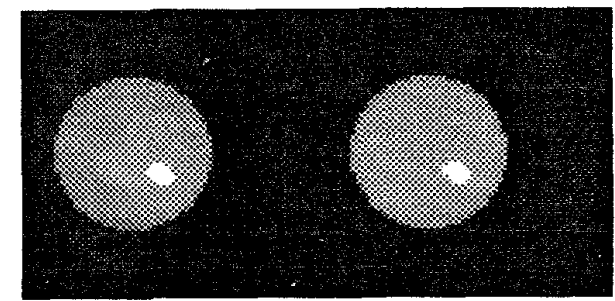

Figure 4: Joint estimation initialized with mismatched MRI. (a). left: reconstructed image, (b). right: estimated labels

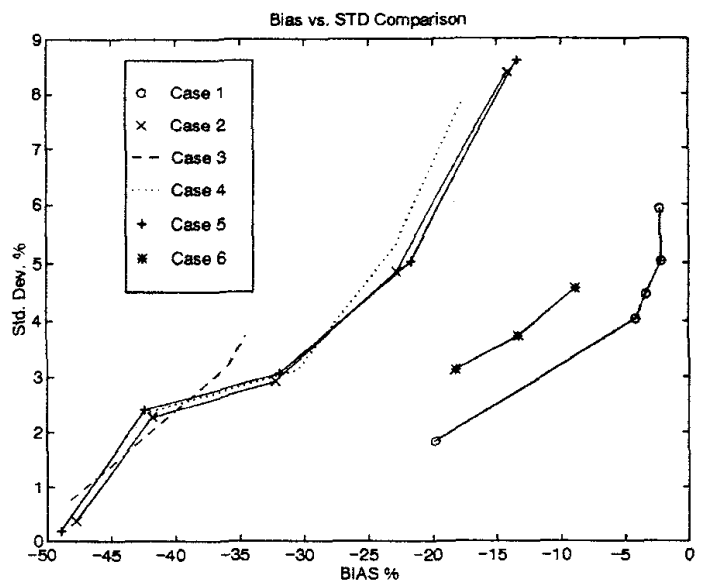

Figure 5: Bias vs. STD in terms of the concentration of the given ROI.

$f_{c}=0.4,0.6,0.8,1.0$ as the following:

$$
\frac{\operatorname{sinc}^{2}\left(f / f_{N}\right)}{\operatorname{sinc}^{4}\left(f / f_{N}\right)+\left(\frac{f}{f_{c} f}\right)^{8}} .
$$

$f_{N}$ corresponds to 0.5 cycles per detector bin(Case4). If MRI information has some mismatch, such as the missing ROI in case 5, fixed application of this MRI information will give the artifact - blurring in the reconstructed SPECT image, so the performance is as poor as case 2 . When the joint estimation is initialized with mismatched MRI anatomical information, it is able to partially recover the region and prevent blurring across the region boundaries, so that the estimation performance (case 6) of concentration in that region is better than case 2,3 and 4 . From the curve we see that case 1 and case 6 are closer, which shows the robustness of the joint estimation.

\section{SUMMARY}

We have shown that the joint estimation approach preserves the benefit of applying the correlated anatomical side information, so the estimation bias and variance are reduced. It can also reduce the influence from the mismatched region information. So the joint estimation approach has the potential of robustly incorporating MRI side information.

In our preliminary study, the $L(1 ; \tilde{1})$ is established by human observation, and simple pixel-by-pixel threshold segmentation is assumed. For more robust application of the joint estimation, an automatic and more systematic method has to be developed in future study. We have also observed that in joint estimation, the convergence is sensitive to the $\left(\beta_{1}, \beta_{2}\right)$ pair, i.e. there exist a range of $\left(\beta_{1}, \beta_{2}\right)$ which will lead the estimation converge to a converge to a local maximum close to the global maximum, otherwise the estimation of both $\hat{\lambda}$ and $\hat{l}$ will converge to a local maximum far from the global maximum. We think that is related to the discreteness of the label variables.

\section{REFERENCES}

[1] X. Yan, R. Leahy, "MAP Image Reconstruction Using Intensity and Line Processes for Emission Tomography Data" SPIE: Image Processing Algorithms and Techniques II, vol. 1452, pp. 158-168, 1991.

[2] S. Geman, D. Geman, "Stochastic Relaxation, Gibbs Distribution and the Bayesian Restoration of Images", IEEE Trans. on Pattern Analysis and Machine Intelligence, Vol PAMI-6 No.6 Nov. 1984.

[3] J. Fessler, N. Clinthorne, L. Rogers, "Regularized Emission Image Reconstruction Using Imperfect Side Information", IEEE Trans. on Nuclear Science, Vol. 39, pp. 1464-1471, No.5, Oct. 1992.

[4] V. Johnson, J. Bowsher, R. Jaszczak, and T. Turkington, "Analysis and Reconstruction of Medical Images Using Prior Information", Discussion Papers Institute of Statistics and Decision Sciences, Duke University, 1993.

[5] J.A. Fessler, A.O. Hero, "Space-Alternating Generalized Expectation-Maximization Algorithm", IEEE Trans. on Signal Processing Vol. 42, No. 10, Oct. 1994

[6] K. Lange and R. Carson, "EM Reconstruction Algorithms for Emission and Transmission Tomography", Journal of Computer Assisted Tomography, 8(2): 306316, April 1984.

\begin{tabular}{|c||c|c|c|c|c|}
\hline$p(l \mid l)$ & $l=0$ & $l=1$ & $l=2$ & $l=3$ & $l=4$ \\
\hline$l=0$ & 1.00 & 0.00 & 0.00 & 0.00 & 0.00 \\
\hline$i=1$ & 0.00 & 1.00 & 0.00 & 0.00 & 0.00 \\
\hline$l=2$ & 0.00 & 0.00 & 1.00 & 0.00 & 0.00 \\
\hline$l=3$ & 0.00 & 0.00 & 0.00 & 1.00 & 1.00 \\
\hline
\end{tabular}

Table 1: Ideal probability distribution of $P\left(\tilde{l}_{k} \mid l_{k}\right)$. 